\title{
BIM 技术在幕墙设计中的应用研究
}

\author{
姚 硕* \\ 沈阳远大铝业工程有限公司，辽宁 110027
}

\begin{abstract}
摘 要: 随着我国生产力的不断提高, 我国社会经济发展越来越迅速, 城市大规模的建设也随之增多。伴随着越 来越多超高层建筑的出现, 形态也跟着变的多种多样, 而外幕墙结构的变化也随着日益复杂。在今天三维技术应用日 趋成熟, 幕墙以及BIM及时也在不断的发展, 也在建筑行业引起了一场变革。本文主要分析了 BIM技术在幕墙设计中 的应用，研究分析BIM技术在幕墙设计中所存在的问题和建议，为今后BIM技术在我国的发展推广应用提供参考。
\end{abstract}

关键词：BIM技术；幕墙设计；应用研究；

\section{Application of BIM Technology in Curtain Wall Design}

\author{
Shuo Yao* \\ ShenYang YuanDa Aluminium Industry Engineering Co., Ltd., Shenyang 110027, Liaoning, China
}

\begin{abstract}
With the continuous improvement of China's productivity, China's social and economic development is getting faster and faster; and the large-scale urban construction has also increased. With the emergence of more and more super highrise buildings, the shape has also become more diverse, and the changes in the structure of the outer curtain wall have become increasingly complex. Today, the application of 3D technology is becoming more and more mature; curtain walls and BIM are constantly developing in time, which has also caused a change in the construction industry. This paper mainly analyzes the application and existing problems of BIM technology in curtain wall design and puts forward some suggestions to provide a reference for the future development and application of BIM technology in China.
\end{abstract}

Keywords: BIM technology; curtain wall design; application research

\section{一、引言}

在社会高速发展中, 城市在不断发展壮大, 随着城市大规模建设的需要, 人们对建设的外观要求也在不断提高, 从而促使建筑行业在建设中不断的出现异形建筑。这种建筑造型复杂, 外观华丽, 以钢为主要结构作为支撑的玻璃幕 墙在建筑中应用的范围越来越广泛。幕墙对于建筑的设计和施工技术来说需要具备更高的要求, 传统的二维软件根本 无法做到这些工程的设计要求, 需要靠三维模型完成, 于是BIM技术走到了建筑行业的视野中 ${ }^{[1]}$ 。如何将BIM技术应 用在幕墙设计中变成了至关重要的部分, 因此BIM技术在幕墙设计中得到了较为广泛的应用, 也为我国幕墙设计的发 展奠定了基础。

\section{二、BIM 技术简介}

BIM技术的英文全称是Building Information Modeling, 也就是建筑信息化模型。BIM技术是在工程设计中用来进 行建造管理和数据化的工具, 以建筑工程中的各项数据作为模型的基础, 进而建立出建筑模型; 通过信息技术仿真 模拟处建筑物的各项真实性信息, 让技术人员可以对各种建筑的信息做到正确理解和应对各种情况, 为设计团队以及 建设团队提供工作的基础信息。BIM模型就是以用BIM技术所模拟建造出来的虚拟模型, 它囊括了建筑物内的所有信 息。为整个建造提高工作效率，将成本减少到最低并且会缩短建造工期等方面。BIM技术具有可视化、模拟分析、多 专业协同设计、修改与图纸的生成等特点。在针对多种设计方式时, 通过可视化这一特点可以更直观有效的进行方案 对比, 选择出最合适的设计方案。

\section{三、BIM 技术在幕墙设计中的应用优势}

(一) 可视化

*通讯作者: 姚硕, 1980 年 11 月, 男, 汉族, 辽宁沈阳人, 就职于沈阳远大铝业工程有限公司, 工程师, 专科。 研究方向：机电工程专业。 
在建筑设计中模型的建立过程主要是施工图和加工图的绘制过程, 跟建筑相关的图纸只是用线条的形式, 将建筑 的构造描绘出来。普通的建模软件最多只能做到表现出这个建筑的外观效果, 更建筑物有关的内部结构。比如说线 路、水暖管道及内部构造等等, 没有办法以三维模型的形式呈现出来, 只能靠专业的设计师根据需要在图纸上描绘来 设想 ${ }^{[2]}$ 。这种设计方式不仅没有办法仔细观察研究建筑内部各单位之间的关系, 还经常会因为图纸出现错误问题。通 过BIM技术的应用不但能模拟出建筑三维形式的外部模型, 还可以将建筑内部的各种构造、线路等以三维形式呈现在 面前, 在设计的过程中可以随时查看幕墙设计的效果, 做到设计、生产和安装的高度协调, 在幕墙设计上更是可以完 全呈现出幕墙的细节问题，即使是包裹幕墙的构件在三维模型上的表达也十分清晰。

\section{(二) 模拟分析}

在模拟分析上, $\mathrm{BIM}$ 技术跟以前的二维技术 $\mathrm{CAD}$ 相比差距更是巨大。 $\mathrm{CAD}$ 主要设计的是图纸, 而 $\mathrm{BIM}$ 技术则是直 接设计模型。 $\mathrm{CAD}$ 的设计图中每一个地方都是单独存在, 无法出现结合的情况, 一旦其中一张图出现问题, 甚至可能 引发相关图纸出现问题, 修改起来需要大量的时间; 而BIM技术直接将图纸变成数据构建模型, 能够从不同的角度对 模型进行观察，及时某处图纸出现了问题进行修改，其他图也会随着自动更新。二维技术在绘制图纸上需要应专门的 软件进行分析计算, 即便是这样设计出的模型仍然存在模型粗粘, 容易出现误差等问题, 而且在建模时需要的时间也 存在过长的问题 ${ }^{[3]}$ 。而通过BIM技术, 可以将绘图、建模计算分析两个要分开进行的工作融合在一起。只要将模型存 人到软件中, 就能直接对模型各项数据进行分析检验, 不仅可以节省大量时间, 还能完善设计方案中的不足之处, 让 数据更加准确无误。

\section{(三) 工厂加工}

由于在幕墙建筑工程中的材料需要从工厂加工, 并且数量庞大; 加上很多材料的加工难度较大周期较长, 工厂需 要派专门的工作人员打印图纸, 以及对图纸进行核对。耗费了大量的人力、物力、财力跟时间, 哪怕是再仔细都容易 出现错误。通过BIM技术来定制幕墙所需材料的批量, 快速建立三维模型, 降低了加工中的图纸数量, 再提高工厂效 率的同时还能为工厂带来极大的成本节约。

( 四) 现场安装

通过BIM技术进行三维模型的建立，可以帮助现场安装时精准定位，提供准确的数据信息。通过数据定位将安装 的偏差控制在允许范围内，这让现场安装的进度得到了快速、可靠的保障，如图1所示。

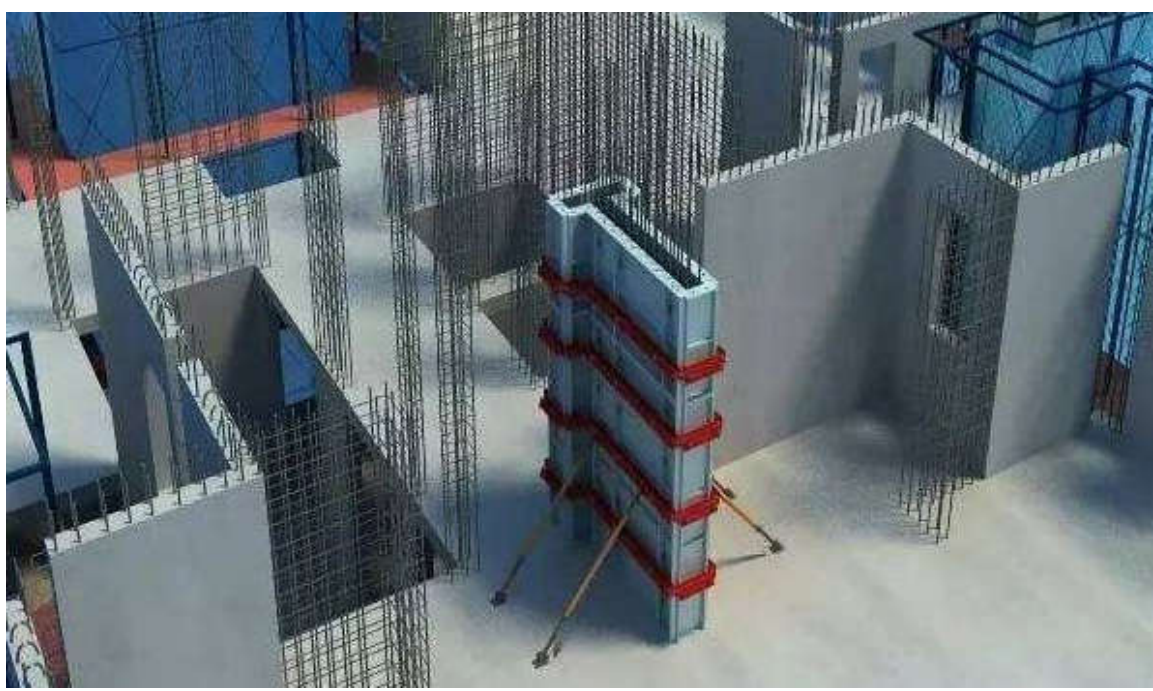

图1 BIM技术模拟图

\section{四、BIM 技术的应用}

当前我国幕墙建设以单元幕墙和框架幕墙为主，其中单元幕墙可提出独立的幕墙单元。每个单元的材料都需要在 工厂独立加工完成, 工厂按照需求生产组织构件, 当建筑主体完成施工后再运到现场进行组装。框架幕墙则是工厂按 照流程跟计划, 根据设计图纸在现场进行组装, 在过程中需要对材料进行二次加工或修复。由于建筑主体的建造跟单 元幕墙可以同时进行, 为了加快进度和提高工作效率, 大多数的建筑工程在建造时都会选择单元幕墙, 而BIM技术又 是单元幕墙的重要工具, 利用BIM技术对单元幕墙进行设计 ${ }^{[4]}$ 。国内在进行幕墙设计时大多数依赖于CAD软件进行二 维图纸制作, BIM技术只会在复杂的设计方案中应用, 比如说双曲弯扭的截面就无法通过 CAD制图进行绘制, 只能通 
过BIM技术强大的三维功能根据参数进行建模, 再在模型基础上对其进行设计更改, 按照幕墙实际的需求进行分格, 最后从三维模型中拆分出各个板块直接生成加工图纸, 流畅完美的表达出设计师想要的效果材料, 以确保指导加工保 证幕墙安装完成。

\section{(一) 曲面设计优化}

通常情况下建筑师对于建筑外形的设计停留在理论性阶段, 很多工程需要的曲面材料是无规则, 只有获得具体 的数据才能进行。用普通的软件很难详细的绘制出外形得到数据, 更不用说对设计进行深化设计, 进行更深方面的 改善。利用好BIM技术的三维功能, 在幕墙设计时可以按照建筑师的设立想法建立出想要的模型, 把理论变成实际模 型，再计算出所需材料的详细数据。比如说鸟巢中的建设就运用到了该技术进行设计，如图2所示。

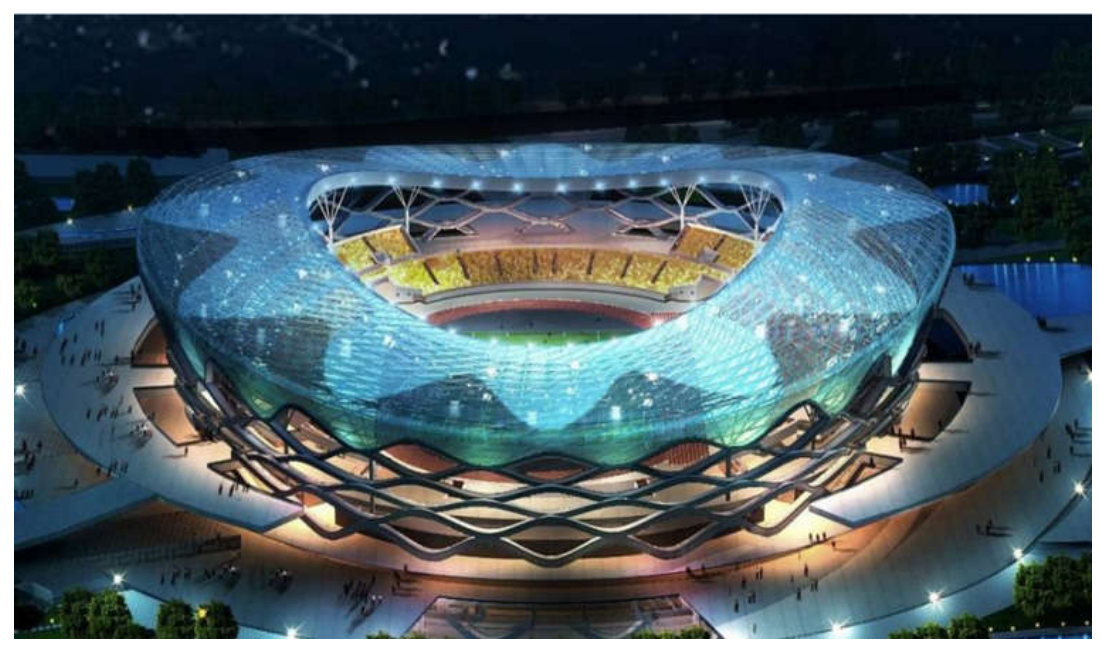

图2 BIM技术的曲面设计应用

\section{(二) 方案制定}

制造出了模型后, 需要在现有的模型基础上进行细化, 也就是把外立面按照幕墙的面材材质的不同进行分格。在 幕墙设计时用BIM技术协助设计师进行数据化的分格, 按照设计师的需求通过数据化控制幕墙的分格, 准确无误的表 达出设计师的理念, 帮助设计师在完成设计想法的同时, 在最大范围内减少制造成本; 在不改变设计师的想法同时, 对曲面玻璃进行平面化降低生产时的难度，帮助工人准确安装减少失误，从而达到减少建造成本的想法 ${ }^{[5]}$ 。

（三）幕墙的建模

根据设计师制造出的图纸和幕墙公司的样图，快速建造出三维模型，将建筑的真实表现状况展示出来，完成基础 的建模步骤, 也可以通过与其他专业模型结合检查幕墙设计中存在的问题。在用BIM技术建造的三维模型的基础上, 对外幕墙、外幕墙的支撑结构以及其他相关进行检查, 快速发现问题, 并在第一时间改变缺陷解决问题 ${ }^{[6]}$ 。通过幕墙公 司的样图和前期制作出的幕墙基础模型, 将模型参数化, 提取出工厂加工、安装时所需要的数据; 再根据幕墙公司的 需求制造出加工图、定位图, 既能保证设计师对幕墙外形的要求, 又能保证再安装施工阶段不对建筑形体进行改变。

( 四 ) 3D模拟碰撞

工作人员在安装施工的过程中往往会遇到大量的构建碰撞问题。传统的2D平面图纸不能对个体和系统进行试验, 并且存在设计人员所忽略掉的碰撞问题。BIM技术则拥有可视化功能, 能够对施工中的碰撞问题进行检测, 之后再进 行优化, 防止各种碰撞情况的出现, 避免施工工程中因为错误造成的不必要损失。促使工程质量的提高, 还能解决业 主临时添加的要求进行有效沟通。

(五) 4D模拟检测、5D工程造价控制和6D综合

$4 \mathrm{D}$ 模拟检测是在 $3 \mathrm{D}$ 的基础上进行, 主要的作用是研究目前方案的可行性, 通过模拟对施工时间和施工工作进行 合理的安排。4D模拟检测的主要作用体现在防止施工中出现的意外, 设计人员和管理人员可以将实际遇到的问题, 通 过BIM技术建造的模型进行研究模拟, 最后制造出合理的方案解决问题。5 $\mathrm{D}$ 主要是在工程造价方面, 建造幕墙过程中 的预算是一件很麻烦的统计工作, 没有BIM技术去收集信息, 很难完成庞大的预算计算工作, BIM技术能对整个工程 所发生的变化进行检测并完成计算, 将幕墙建设预算降到最低 ${ }^{[7]}$ 。6 $\mathrm{D}$ 是综合性的模拟, 主要内容包括: 对幕墙抗风性 能的研究, 对幕墙抗震心内的研究, 对幕墙内空气流通试验的模拟, 对于幕墙水密性能的研究, 对于幕墙节能的模拟 试验。检查幕墙是否到达建筑规范要求, 确保幕墙建筑的最大效益, 将幕墙的性能发挥到最大程度。 


\section{（六）构件加工图}

根据定位图和数据, 将模型直接导出材料加工图纸，与承接加工任务的工厂对接进行材料的加工。在BIM模型的 基础上, 可以对现场测量的数据进行快速分析, 自行对不同偏差的情况进行判断, 将效率极大地提高。同时还可以通 过现场的测量数据, 快速修正设计模型出现的问题, 提取加工图获取详细的理论数据。哪怕是在设计出现的偏差情 况, 也能通过快速设计进行改变, 在最短的时间内将构件运至现场, 减少了时间的浪费。在进行BIM模型拼装时, 对 于偏差大的情况，可以通过测试手段和设备自动生产所需的单元板块，在理论模型中进行预拼装。

（七）通过简易动画指导安装

可视化的模型是指导现场安装的重要参考，通过模型进行的施工顺序指导，在BIM制造的现场模型上进行分析模 拟，给予现场工作人员的演示。

\section{（八）数据信息的共享}

BIM模型中包含了大量的数据信息, 这些信息可以在数据后台的数据库进行共享, 负责建筑的各个部门人员可以 在同一个平台上实现数据的分享, 方便不同部门间的沟通和协商解决问题。BIM在幕墙施工的工程中, 能够根据原有 的数据进行优化, 对工程中存在的问题和错误, 进行及时的调整。BIM系统还会对数据信息进行保存, 通过信息共享 如果施工中有一方对数据产生异议, 可以及时作出合理的调整。在传统幕墙建筑的施工过程中, 不能将数据信息共 享, 施工人员在进行协商时还会耽误工程的进展 ${ }^{[8]}$ 。BIM就可以极大的减少施工现场对测绘的工作量, 最大程度的控 制施工质量。在BIM模型的基础上还可以通过其他软件进行信息整合, 甚至能够做出动画进行观赏, 以最客观的程度 展现出组装加工的工程。

BIM技术在幕墙设计中分为两个阶段: 一是初始阶段, 以传统的项目管理为主, 用BIM技术作为辅助功能, 这个 阶段的BIM技术往往是根据工程的进展, 选在一项内容进行应用, 不会改变幕墙设计、施工等工作流程。二是高级阶 段, 高级阶段的BIM技术在建造幕墙的各个阶段提供解决方案，参与各项数据的收集整合工作，以确保数据信息的准 确, 如图3所示。

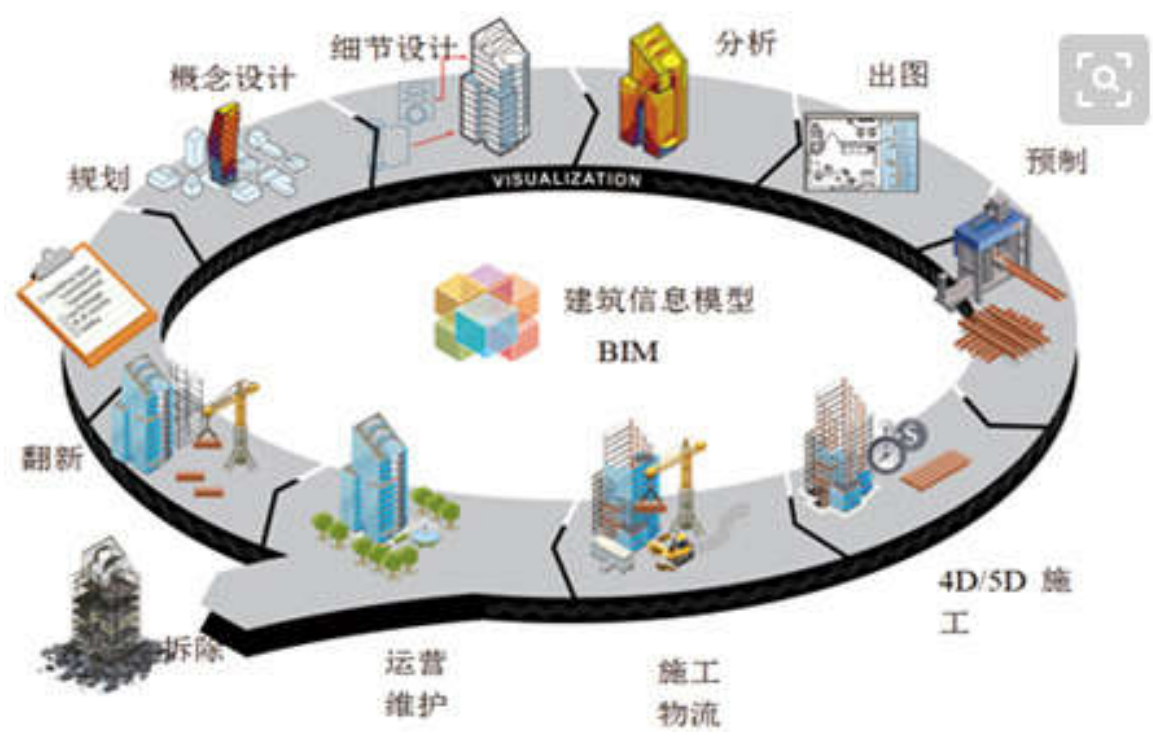

图3 BIM技术在幕墙设计中的流程图

\section{五、BIM 技术的相关应用软件}

(一) 民用建筑: Autodesk Revit

(二) 工厂设计和基础设施: Bentley

（三）专业建筑事务所：ArchiCAD，Revit，Bentley

（四）项目完全异形、预算比较充裕: DigitalProject

\section{六、结语}

综上所述, BIM技术在幕墙设计中有着不可替代的重要地位, 它贯穿整个幕墙的工作, 从最初的设计到最终的安 装完成, 每一步都需要BIM技术的应用。在我国很多的知名建筑中都运用到了BIM技术, 但从广大范围上来看, BIM 技术在我国还处于刚起步的阶段。随着社会建筑的越来越多, BIM技术的发展与普及, 在以后的幕墙设计中, BIM将 
成为幕墙设计工程中不可或缺的一部分。为了将BIM技术更好的应用到幕墙设计中, 还需要我国建筑企业在各方面的 细致管理, 运用最科学的办法将效益最大化。

\section{参考文献:}

[1]王梓涵, 毛锴文, 李国强, 章雨晨, 林王晨, 李国文.BIM技术在如东某医院外科大楼幕墙中的应用 [J]. 价值工程, 2019,38(28):265-269.

[2]宋凌月.浅谈主流BIM软件在幕墙设计中的运用[J].中国建材, 2019(09):144-147.

[3]林锐涁.BIM技术在幕墙设计中的应用 [J].住宅与房地产, 2019(24):162.

[4]宗兆民,王福龙,刘明文,间金輇,鲁晋.BIM技术在中央歌剧院剧场幕墙工程中的应用[J].城市住宅, 2019,26(07):21-23.

[5]佟克龙.BIM技术在仿钻石切面的玻璃幕墙设计中的应用[J].建筑施工, 2019,41(01):166-167.

[6]姚瑞哲. BIM技术在复杂综合体项目施工阶段的应用 [D]. 华南理工大学, 2018.

[7]刘智波,剧小凡.BIM技术在复杂幕墙工程设计及施工中的有效应用[J]. 居舍, 2018(25):16.

[8]葛利龙,葛艳艳.探究BIM技术在幕墙装饰工程中的应用 [J].居舍, 2018(23):55. 